\title{
Les enjeux économiques de la géolocalisation pour les réseaux sociaux numériques.
}

\author{
Marc Bassoni, Ecole de Journalisme et de Félix Weygand, Université de la \\ Communication de Marseille \& IRSIC EA Méditerranée, IRSIC EA 4262 \& Euromed- \\ 4262 (bassoni@ejcm.univmed.fr) \\ Management \\ (felix.weygand@univmed.fr)
}

Les réseaux sociaux numériques (désignés par la suite «réseaux sociaux ») et les usages qu'ils nourrissent ont pris une place significative au sein de la galaxie Internet. $\mathrm{Ce} \ll$ Web social » se caractérise par un développement rapide des audiences et un potentiel «viral» incontestable. Pour autant, le modèle économique fondé sur la gratuité pour les utilisateurs des réseaux « leaders »n'est pas encore solidement établi. La conversion durable et pérenne des audiences en profit n'est pas totalement acquise.

L'objectif de cet article est double : d'abord, mettre à plat la problématique complexe de cette conversion en la situant dans le contexte économique des marchés «multiversants »(1) (modèle économique de référence des réseaux «leaders»); ensuite, découvrir comment le «Web social » et le «Web mobile» se rencontrent. Nous expliciterons la condition sous laquelle la géolocalisation pourrait conforter à terme la rentabilité économique du «Web social »; un «pure player» du réseau social gratuit et géolocalisé sur l'Internet mobile, la société $a k a$ 'aki, sera pris comme exemple.

\section{Marché multiversant et course à l'audience}

Les réseaux sociaux constituent une famille de plates-formes très hétérogène. Si certains, parmi eux, demeurent «confidentiels », ou confinés au sein de communautés très limitées, d'autres, en revanche, agrègent des audiences très vastes. Quelle que soit leur taille, ces réseaux font exploser les démarcations traditionnelles entre la communication interpersonnelle (privée) et la communication publique et doivent leur succès à leur 
« horizontalité »; les « découvertes d'information » qu'ils rendent possibles s'opèrent via le réseau des proches. De par le monde, 940 millions d'internautes sont membres d'au moins un réseau social (d'après InSites Consulting, janvier 2010) ; 51 \% utilisent Facebook, 17 \% utilisent Twitter. Le 5 mars 2010, ce dernier a franchi le cap symbolique des 10 milliards de messages («tweets ») postés depuis la création du réseau (en 2006).

Si certains, notamment les réseaux dédiés à la rencontre amoureuse comme Meetic, font payer l'utilisateur final, les grands réseaux sociaux fondés sur la gratuité fonctionnent économiquement selon le principe des marchés multiversants: le bien, ici un service immatériel d'information ou de mise en relation, est vendu à différentes catégories de consommateurs reliées entre elles par l'usage de ce bien (ou de ce service); le fournisseur peut arbitrer la structure des prix entre ces catégories. Ainsi le (ou les) versant(s) qui seront peu facturés, voire gratuits, seront "subventionnés" par les versants plus facturés (Rochet \& Tirole, 2005). Des externalités de réseau ${ }^{(2)}$ s'exercent entre les groupes d'agents mis en relation par le marché sur ses différents versants (Katz \& Shapiro, 1994 ; Economides, 1996 ; Pénard \& Suire, 2008). Dès lors qu'une masse critique d'utilisateurs est atteinte sur un versant, un cercle vertueux se crée qui augmente l'utilité pour les utilisateurs de l'autre versant et ainsi leur nombre et leur consentement à payer ; la gratuité sur le premier versant permet d'atteindre rapidement la masse critique et de lancer le cercle vertueux (Weygand, 2009). Cette gratuité pour l'usager final doit être subventionnée par les autres versants grâce à un modèle économique fondé essentiellement sur l'économie de l'attention ${ }^{(3)}$. Les grands réseaux sociaux exploitent ainsi ces externalités : plus les utilisateurs sont nombreux à dévoiler leurs préférences, plus les annonceurs sont appâtés par cette audience qualifiée ; réciproquement, plus ces revenus s'étoffent, plus les réseaux se développent, offrent des services attractifs et gagnent de l'audience (Rebillard \& Smyrnaios, 2010). Alors même que la conquête de la masse critique d'abonnés gratuits ne rapporte rien mais génère des coûts très importants, rien ne vient pour autant garantir la transformation de cette «attention» en revenus et en profits.

\section{Pression des pairs et quête de rentabilité}

Cette question de la création de valeur sur un Internet où, apparemment «tout est gratuit » (Gensollen, 1999), est posée dès le début de l'expansion commerciale sur le réseau; les entreprises qui ont pu y répondre avant l'explosion de la bulle à partir de mars 2000 ont vu 
leurs modèles d'affaires devenir structurants au sein de l'économie numérique (Mahadevan, 2000). Depuis le redémarrage de celle-ci à partir de 2004 les nouveaux venus, dont les réseaux sociaux, se sont construits en référence à ces modèles (Garonne \& Weygand, 2010), sans que l'imitation ne garantisse le succès dans le nouveau contexte. Ainsi, au premier trimestre 2010, sur le seul marché américain, Facebook accueille plus de bannières publicitaires que Yahoo! (176,3 milliards de bannières pour Facebook, contre 131,6 milliards pour Yahoo!, d'après comScore Ad Metrix). Toutefois la performance en termes de revenus est décevante pour Facebook car, pour s'assurer une percée sur le marché du «display» (bannières), le réseau casse ses prix et, pour un annonceur, le coût des bannières sur Facebook est largement inférieur à ce que facture Yahoo! Pour Facebook, l'audience étant désormais acquise, il importe de faire évoluer la politique tarifaire et, surtout, de transformer les sources de revenu (Laugée, 2010). Cette préoccupation est encore plus forte chez Twitter. En effet, à la fin de l'année 2009, le réseau-star de micro-blogging ne doit son développement qu'aux injections de fonds consenties par le capital-risque. Sa quête de revenus est impérieuse. Déjà, le réseau commence à commercialiser des flux de données à des services de recherche (Google, Bing) ; depuis mars 2010, il teste également un système de «tweets sponsorisés », bâti sur le modèle des liens publicitaires de Google.

Chez Facebook, la quête du « Graal » est relancée en avril 2010 avec le lancement d' «Open Graph » puis, en août, avec l'inauguration de «Places ». La première innovation met en avant une nouvelle fonctionnalité (bouton «Like ») qui permet à chaque internaute d'indiquer à ses « amis » le contenu attractif ou séduisant d'un site-tiers, partenaire du réseau social. La seconde, embarquée sur un smartphone, permet de savoir où se trouvent physiquement ses «amis » et réciproquement de leur signaler en temps réel le lieu où l'on est. Ces fonctionnalités «re-territorialisent» et donnent corps à ce que D. Fletcher appelle «la publicité sous pression des pairs » (Fletcher, 2010, p. 22). «Les professionnels savent cela depuis très longtemps - précise Sh. Sandberg ${ }^{(4)}$, Directrice de l'exploitation chez Facebook -, les gens sont d'autant plus enclins à faire quelque chose que cela leur a été recommandé par un ami » (citée par Fletcher, 2010, p. 22).

Couplé à la géolocalisation, le marketing (qualifié de « viral », «tribal », « communautaire », etc.) est ainsi en train de se rénover grâce aux potentialités que recèlent les réseaux sociaux (Le Rouzic, 2010). Comme le dit le PDG de Facebook aux annonceurs : « Nous allons aider vos marques à faire partie des conversations quotidiennes » (Ducourtieux \& Girard, 2007).

Ce mouvement réintroduit fortement la géographie dans le commerce et le marketing électroniques: pour les «gens de marketing», les annonceurs et les marchands, connaître 
finement et en temps réel les goûts des consommateurs, pénétrer leur intimité, filtrer et guider leurs choix «sous pression des pairs » sont d'autant plus utiles qu'ils savent où se trouvent ces consommateurs pour mettre en avant les lieux de vente et de consommation des produits et services dont ils ont repéré ou éveillé le désir.

Aux Etats-Unis en 2009, pour le seul marché du « display » (soit 8,7 milliards de dollars), la publicité géolocalisée représentait un peu plus de $12 \%$. Cette part pourrait grimper à $15 \%$ en 2013 ; la publicité géolocalisée à l'échelle d'une ville, et non plus seulement au niveau d'une région, représentant alors $30 \%$ de ce marché (Le Journal du Net, avril 2010). Quant à la publicité (liens sponsorisés et «display») sur l'Internet mobile, elle devrait passer de près d'un milliard de dollars en 2009 à 4,2 milliards de dollars en 2015 (Le Journal du Net, janvier 2010).

\section{Géolocalisation, réseaux sociaux, web mobile : l'exemple d'aka'aki}

$A k a^{\prime} a k i^{(5)}$ est typique de l'hagiographie des start-up de l'Internet: un sujet de thèse d'étudiants berlinois en 2007 que ceux-ci ont transformé, en avril 2008, en entreprise. En mars 2010, cette dernière a annoncé avoir passé le cap des 500000 membres (principalement en Allemagne et en France), en ligne en moyenne 30 minutes par jour sur son service où s'échangent 300000 messages quotidiens. Le grand fonds allemand de capital-risque, Creathor venture, la soutient depuis le début et a été rejoint, fin 2009, par Innovacom, le fonds de capital-risque de France Telecom Orange $e^{(6)}$.

Le principe est classiquement celui des réseaux sociaux : un service Web permet à chaque utilisateur de créer son «profil » en ligne, de décrire ses caractéristiques, ses préférences et éventuellement ses motifs de converser ou de rencontrer d'autres utilisateurs. A partir de là, il accède aux profils des autres utilisateurs qu'il peut trier par pays, ville, âge et sexe afin d'entrer en interaction, de leur envoyer des invitations à devenir « ami » et de recevoir les leurs. Un système de messagerie et divers dispositifs permettant de manifester, publiquement ou à son seul réseau d'« amis », ses variations d'humeur, de goût, d'opinion, etc., font le reste. Le tout peut être interconnecté avec Twitter, et depuis peu avec Facebook au travers d'un développement que l'on évoquera plus loin.

Aka'aki n'est qu'incidemment un site Web; il s'agit avant tout d'une application dont le terminal désigné est le téléphone mobile, ou plus réellement le «smartphone »; le service a connu une accélération à partir de sa disponibilité sur l'iPhone en février 2009. Son 
fondement est un puissant système de géolocalisation utilisant les réseaux de téléphonie mobile ainsi que les fonctions GPS, Bluetooth et Wifi des terminaux. L'application aka'aki du téléphone mobile repère ainsi qui sont les autres membres du réseau social qui sont « tout près », « à une distance de marche », « en ville » et «dans la région »; grâce à cette indication de proximité, l'utilisateur peut entrer en contact virtuellement puis, très vite, rencontrer réellement les autres membres.

L'application mobile se télécharge gratuitement mais supporte quelques insertions publicitaires ; comme pour les grands réseaux sociaux fondés sur la gratuité, le modèle économique d'aka'aki est encore peu stabilisé et, pour l'heure, cette entreprise fonctionne essentiellement en consommant les fonds levés auprès des sociétés de capital-risque. Plus encore que pour les autres réseaux sociaux, la masse critique permettant d'amorcer le cercle vertueux des effets de réseaux croisés est importante et difficile à atteindre pour aka'aki. Pour l'utilisateur final, la différence qui donne à ce service une utilité supérieure à celle d'un autre réseau social c'est la possibilité de rencontres réelles autorisées par la géolocalisation et la mobilité. Pour que ces rencontres aient effectivement lieu assez souvent pour rendre le service attractif, il est impératif qu'une certaine densité géographique d'utilisateurs soit atteinte ; face à cela, les 500000 membres dispersés d'aka'aki sont une performance dérisoire.

La seule gratuité ne suffisant pas à atteindre cette masse critique, aka'aki doit trouver de nouveaux usages attractifs et faire fonctionner à son profit la «pression des pairs » exercée via les réseaux sociaux leaders que sont Facebook et Twitter. Ces développements sont en cours puisque l'entreprise de Berlin vient de lancer, fin août 2010, un jeu mobile géolocalisé (lift loft) qui supporte de la publicité, qui est disponible pour les seuls utilisateurs d'iPhones et qui exige la possession d'un compte Facebook.

\section{Conclusion}

Ce virage récent, opéré par aka'aki, montre bien que la conversion pérenne des audiences en profit emprunte une voie étroite et incertaine. La start-up berlinoise envisage en effet de s'adosser à Facebook pour promouvoir un jeu, a priori plus attractif et plus générateur de trafic que les usages traditionnels. Partant, aka'aki s'en remet au modèle de publicité «sous pression des pairs » exploité par la firme de Palo Alto ; ce modèle, pour intéressant qu'il soit en termes de ciblage et de filtrage, n'en est pas moins fragile: comment réagiront les utilisateurs de ces plates-formes lorsque ledit ciblage deviendra, du fait même de son 
efficacité, trop visible et trop « intrusif »? En France, le buzz qui accompagne la naissance de seppukoo.com (2009) - réseau « antisocial » qui promeut le « suicide » des identités virtuelles - est un premier signe de défiance ; l'initiative canadienne du «Quit Facebook Day », lancée le 31 mai 2010, constitue un autre signal d'alerte. Cette sensibilité au risque croissant de contrôle social n'est pas négligeable (Proulx, 2011); nous pensons qu'elle va s'exacerber au rythme de l'exploitation des promesses offertes par la géolocalisation (cf. les récents déboires du programme Google Street).

Confronté à ce «talon d'Achille », les réseaux sociaux numériques peuvent emprunter des voies alternatives. La première à l'initiative de ses entrepreneurs qui feraient apparaître un nouveau versant rémunérateur, notamment par un prélèvement sur les opérateurs de télécommunications ou de systèmes d'exploitation mobiles. La seconde sous l'action des utilisateurs eux-mêmes, ou du moins d'une minorité agissante en leur sein qui, sans renoncer à la gratuité des nouvelles socialisations permises par le Web, refuseraient le «pillage » de leur attention et de leur intimité pour y substituer un modèle coopératif (Cardon, 2010).

\section{Notes}

(1) La notion de «marché multiversant » est une généralisation de celle de marché «biface » (ou «à double versant», traduction de l'anglais double sided market ; cf. Rochet \& Tirole, 2005).

(2) Un bien ou un service présente des externalités de réseau lorsque l'utilité d'un consommateur augmente avec le nombre de consommateurs du même bien ou du même service.

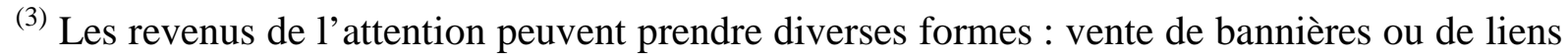
sponsorisés, commission sur ventes électroniques, revente de données sur les parcours et profils des internautes issues du data mining, ...

(4) Sh. Sandberg fut, pour Google, la «cheville ouvrière » du très efficace AdWords qui permet aux annonceurs d'acheter des insertions publicitaires en fonction des requêtes des usagers.

${ }^{(5)}$ Les informations concernant $a k a$ 'aki, à l'exception des informations sur les investisseurs, sont issues du blog (http://blog.aka-aki.com/), du site de son jeu lift loft (http://games.akaaki.com/news-english.html), du site de l'entreprise (http://www.aka-aki.com) ainsi que de l'utilisation expérimentale de son service mobile par les auteurs au cours de l'été 2010 (http://m.aka-aki.com).

${ }^{(6)}$ Les informations sur les investisseurs sont issues du Journal du Net («L'Allemand aka'aki lève plusieurs millions d'euros », 22 octobre 2009) et des sites Web de Creathor Venture (www.creathor.de) et Innovacom (www.innovacom.com).

\section{Références}

Cardon D., La démocratie Internet. Promesses et limites, Seuil, Paris, 2010. 
Economides N., «The Economics of Networks », International Journal of Industrial Organization, 14, 1996, pp. 673-699.

Garonne Ch., Weygand F., « Transformation of Internet-based Companies’ Business Models : Ten Years after the Dot.com Burst » in The Media Industries and their Markets, Quantitative Analyses, Palgrave Macmillan, 2010, pp. 26-48.

Gensollen_M., «La création de valeur sur Internet », Réseaux, n 97, 1999, pp.15-76.

Katz M.-L., Shapiro C., « Systems Competitions and Network Effects », Journal of Economic Perspectives, Vol. 8, n 2, Spring 1994, pp. 93-115.

Laugée F., « Marques de média et médias de marque », La Revue européenne des médias, $\mathrm{n}^{\circ}$ 14-15, printemps-été 2010, pp. 50-56.

Le Rouzic S., «L'utilisation des réseaux sociaux en études marketing », juin 2010. En ligne sur http://www.slideshare.net/LaRecherche2.0. Consulté le $1^{\text {er }}$ juillet 2010.

Mahadevan B., «Business models for Internet-based E-commerce : an Anatomy », California Management Review, 42, 4, Summer 2000, pp. 55-69.

Pénard Th., Suire R., «Economie de l'Internet: une économie d'interactions sociales », Revue française d'économie, Vol. XXII, n 3, 2008, pp. 151-188.

Proulx S., «L'usage des réseaux sociaux numériques: une intériorisation douce et progressive du contrôle social », Hermès, 2011 (dans ce même numéro).

Rebillard F., Smyrnaios N., «Les infomédiaires, au cœur de la filière de l'information en ligne », Réseaux, n 160-161, 2010, pp. 164-194.

Rochet J.-Cl., Tirole J., « Two-Sided Markets : a Progress Report », IDEI Working Papers $\mathrm{n}^{\circ}$ 275, November 2005. En ligne sur http://www.idei.fr/doc/wp/2005/2sided_markets.pdf.

Weygand F., «Economie de la « société de l'information ». Quoi de neuf? », TIC \& Société, Vol. 2, n 2, 2009. En ligne sur http://revues.mshparisnord.org/ticsociete/index.php?id=499.

\section{Sources documentaires}

Cardon D., «Pourquoi l'Internet n'a-t-il pas changé la politique ? », Interview par Hubert Guillaud, Internet Actu, le 7 octobre 2010. En ligne sur http://www.internetactu.net. Consulté le 13 décembre 2010.

Ducourtieux C., Girard L., « Le site Facebook vend le profil de ses internautes aux publicitaires », Le Monde, 10 novembre 2007. En ligne sur http://www.lemonde.fr. Consulté le 6 août 2010. 
Fletcher D., «Friends Without Borders », Time, May 31, 2010, pp. 18-24.

Le Journal du Net, «Etats-Unis : le marché de la publicité sur mobile », 25 janvier 2010. En ligne sur http://www.journaldunet.com. Consulté le 5 août 2010.

Le Journal du Net, «Etats-Unis : le marché de la publicité en ligne », 12 avril 2010. En ligne sur http://www.journaldunet.com. Consulté le 5 août 2010. 